\title{
Aberrant Temporal-spatial Patterns to Sad expressions in Major Depressive Disorders via Hidden Markov Model
}

Zhongpeng Dai ${ }^{1,2}$, Siqi Zhang ${ }^{1,2}$, Hongliang Zhou ${ }^{3,4}$, Xinyi Wang ${ }^{1,2}$, Huan Wang ${ }^{1,2}$, Zhjjian $\mathrm{Yao}^{3,4 * *}$, Qing $\mathrm{Lu}^{1,2} *$

1. School of Biological Sciences \& Medical Engineering, Southeast University, Nanjing 210096, China

2. Child Development and Learning Science, Key Laboratory of Ministry of Education, China

3. Department of Psychiatry, the Affiliated Brain Hospital of Nanjing Medical University, Nanjing 210029, China

4. Nanjing Brain Hospital, Medical School of Nanjing University, Nanjing 210093, China

* Correspondences: Professor Qing Lu, School of Biological Sciences \& Medical Engineering, Southeast University, Jiangsu Province, No. 2 Sipailou, Nanjing 210096, China. e-mail: luq@seu.edu.cn and Zhijian Yao, e-mail: zjyao@njmu.edu.cn 


\begin{abstract}
Background: The pathological mechanisms of Major depressive disorders (MDD) is associated with over-expressing of negative emotions, and the overall temporal-spatial patterns underlying over-representation in depression still remained to be revealed to date. We hypothesized that the aberrant spatio-temporal attributes of the process of sad expressions relate to MDD and help to detect depression severity.
\end{abstract}

Methods: We enrolled a total of 96 subjects including 47 MDDs and 49 healthy controls (HCs), and recorded their Magnetoencephalography data under a sad expressions recognition task. A hidden Markov model (HMM) was applied to separate the whole neural activity into several brain states, then to characterize the dynamics. To find the disrupted spatial-temporal features, power estimations and fractional occupancy of each state were contrasted between MDDs and HCs.

Results: Three states were found over the period of emotional stimuli processing procedure. The visual state was mainly distributed in early stage $(0-270 \mathrm{~ms})$ and the limbic state in middle and later stage $(270 \mathrm{~ms}-600 \mathrm{~ms})$ of the task, while the fronto-parietal state remained a steady proportion across the whole period. MDDs activated statistically more in limbic system during limbic state $(p=0.0045)$ and less in frontoparietal control network during fronto-parietal state $\left(p=5.38 * 10^{-5}\right)$ relative to HCs. Hamilton-Depression-Rating Scale scores was significantly correlated with the predicted severity value using the state descriptors $(p=0.0062, r=0.3933)$.

Discussion: As human brain exhibited varied activation patterns under the negative stimuli, MDDs expressed disrupted temporal-spatial activated patterns across varied 
stages involving the primary visual perception and emotional contents processing compared to HCs, indicting disordered regulation of brain functions. Furthermore, descriptors built by HMM could be potential biomarkers for identifying the severity of depression disorders. 


\section{Introduction:}

In major depressive disorder (MDD), patients usually exhibit a mood-congruent processing bias especially toward the processing of negative emotional facial expressions (Stuhrmann, Suslow, \& Dannlowski, 2011). This kind of mood-congruent bias makes MDD patients over-express negative information (Dalili, Penton-Voak, Harmer, \& Munafo, 2015). This phenomenon was characterized with the mechanisms of stimulus processing and specific over-stimulated biological system (Furey, 2011; Roiser, Elliott, \& Sahakian, 2012). The response to negative emotional stimulus in visual areas would be regarded as biomarkers for depression (Furey et al., 2013).

A series of event-related potentials (ERPs) studies have shown that the processing of negative emotional information bias could be a potential phenotype for MDD Harris, \& Williams, 2018). During the process of negative stimulus task, several ERPs waveforms were identified to be associated with MDD. Early P1 (100-150ms) reflecting fast perceptual process and evoked unconscious emotions in MDD. Middle N170 (150-200ms) was regarded as indispensable component of emotional processing. The amplitude of late P300 (250-600ms) was suggested to be a biological marker for pathophysiological mechanisms. According to these founds, one can see that the negative bias for MDD was distributed over various temporal stages following the onset of emotional stimuli.

Since the different temporal stages for the process of the negative emotion were 
closely associated with impaired neural mechanism for MDDs, aberrant spatial patterns underlying different periods deserved further analyses. Based on this, literature reported that the responsiveness of brain regions like amygdala and hippocampus, which were involved in limbic system, was associated with depression severity by utilizing sliding time window algorithm (Bi et al., 2019; Suslow et al., 2010). Previous time frequency analysis in sensor level has found that MDD patients expressed increased activations in occipital lobes when dealing with sad faces (Jiang et al., 2019). Neural activities in prefrontal cortices were hypo-activated in MDD patients under the negative emotional stimulus with dynamic connectivity regression (DCR) algorithm (Bi et al., 2016). Besides, the response of the parietal lobe to sad faces also negatively correlated with disorders severity according to micro-state analysis (Mel'Nikov et al., 2018; Soni, Muthukrishnan, Sood, Kaur, \& Sharma, 2018). Lots of evidences have suggested that MDD patients exhibited the disrupted spatiotemporal specificity when dealing with sad facial stimulus, nevertheless, studies revealing the transient dynamics in large-scale brain networks were still rare in relation to the task. Thus, further exploration is required to probe the rich dynamic dysfunction underlying MDD of during the whole period for stimuli processing.

A novel data-driven algorithm Hidden Markov model (HMM) could be utilized to characterize the dynamics of the temporal-spatial brain patterns in network level. Relative to traditional window-based approaches, it could capture transient neural signals under pure negative emotion in milliseconds for each subject, without 
pre-specifying the window length (Quinn et al., 2018; Vidaurre et al., 2016). Besides, it is convenient for HMMs to generate state-wise mean activation maps in large-scale network via the multivariate observation model, as well as to observe the processing of visual perception, decision making and motor response by the sequential spatiotemporal activation maps. Recent study also showed that impaired brain dynamics could be characterized not only in limited targeted regions but also in the large-scale brain networks via HMMs (Charquero-Ballester et al., 2020). Furthermore, dynamic descriptors such as fractional occupancy inferred from HMMs were found to correlate with symptoms of schizophrenia patients, emerging the potential of the promotion to other psychosis, like MDD (Zhi, Calhoun, Lv, Ma, \& Ke, 2018).

In the present study, we aimed at exploring the abnormal spatiotemporal brain patterns in MDD patients under the negative emotional task. To achieve this, an AE-HMM model was applied on Magnetoencephalogram (MEG) data recorded under the stimulus of sad facial recognition task. MEG with high temporal resolution in milliseconds could be utilized to explore neuropsychological mechanisms of fast neural activity for MDD. The processing of chronological neural activity across visual task was characterized by several brain states whose dynamic descriptors are identifiable for MDD. Furthermore, regression analysis was adopted to explore the ability of these dynamic descriptors to indicate the severity of disorders in MDD. Our study might be a promotion to apply HMM to the field of MDD. We provided a new 

available under aCC-BY 4.0 International license.

perspective to the evolution process of negative emotional stimulus over the visual task especially in MDD patients. 


\section{Methods}

\section{Participants}

One hundred individuals (50 $\mathrm{HC}$ and $50 \mathrm{MDD}$ ) were enrolled in the Nanjing Brain Hospital between October 2011 and June 2016. All individuals were given Mini international Neuropsychiatric Interview (MINI) to exclude potential MDD from HC. After exclusion in clinical judgment (potential bipolar disorders) and imaging quality (excessive head motion and other artifacts), ninety-six individuals (49 HC and 47 MDD) were recruited in this study. They were all matched in gender, age and education (Table1). For MDD, the severity of disease was assessed by professional psychiatrists using Hamilton Depression Rating Scale (HAM-D) and the Diagnostic and Statistical Manual of Mental Disorders, fourth edition (DSM-4). The inclusion criteria were no brain injury, alcohol or drug abuse. All individuals were right-handed and provided with written consent forms. This study was approved by the ethical committee at Nanjing Brain Hospital.

Table 1. Demographic and clinical scores of all individuals

\begin{tabular}{lcccc}
\hline & MDD & HC & $t$ & $p$-value \\
& $\mathrm{N}=47$ & $\mathrm{~N}=49$ & & \\
\hline Gender (male/female) & $24 / 23$ & $25 / 24$ & 0.094 & 0.844 \\
Age (years) & $31.68 \pm 7.54$ & $31.53 \pm 7.40$ & 0.057 & 0.963 \\
Education (years) & $13.57 \pm 5.91$ & $14.47 \pm 4.56$ & 0.112 & 0.828 \\
Score of 17-item HAMD & $21.83 \pm 5.95$ & & & \\
\hline
\end{tabular}

Data were presented as the range of mean \pm standard deviation (two tailed $t$-tests) 


\section{Stimuli and task}

All individuals were engaged in a passively viewing task of emotional faces. Forty sad facial expressions were chosen from the Chinese Facial Expression Video System (Jiang et al., 2019; Jing-Lun, Yao, \& Xie, 2007). Each video was presented lasting for 3 seconds. Then a fixation cross picture was also displayed during the rest. An random inter-trial interval of $0.5 \mathrm{~s}, 1 \mathrm{~s}$, or $1.5 \mathrm{~s}$ followed each facial expression.

\section{Data acquisition and preprocessing}

Task MEG data were recorded with a whole-head CTF275 MEG system (VSM Inc) with a $300 \mathrm{~Hz}$ sampling rate. Individuals were scanned lying in the supine position in a magnetically shield room. When scanned, individuals were required to adjust head positions if their head motions exceeded $5 \mathrm{~mm}$ compared to the initial position. Individuals' structural T1 images were recorded using a 1.5T GE system and 3D gradient-echo pulse sequence.

MEG data were pre-processed using Matlab-based fieldtrip toolbox (Oostenveld, Fries, Maris, \& Schoffelen, 2011). First, Notch filter was used to remove $50 \mathrm{~Hz}$ power line noise. Then, we excluded trials containing excessive variance with large channel jumps based on visual inspection. No significant difference in the number of excluded trials was found between MDD and HC (MDD:6.1 \pm 2.7 ; HC:5.3 \pm 3.2 ). Furthermore, data were decomposed into 275 components using an independent component analysis (ICA) (Jung, Makeig, Westerfield, Townsend, \& Sejnowski, 2001). The 
number of ICA components was equal to the number of MEG sensors and components related to cardiac and muscle artifacts, eye blinks were rejected.

\section{Source reconstruction}

The pre-processed data in sensor space were projected in source space onto a $6 \mathrm{~mm}$ grid by a Linearly Constrained Minimum Variance (LCMV) beamformer (Woolrich, Hunt, Groves, \& Barnes, 2011). The LCMV beamformer normalized grid in MNI space and constructed a realistic head model using participant's structural MRI. A covariance matrix was calculated across all trials using the spatial filters. Subsequently, the spatial orientations of each epoch were rotated in order to maximize the variance. Source activity was estimated by multiplying spatial filters with sensor-level time series across the whole scale. Subsequently, a multivariate symmetric orthogonalization was applied to attenuate the spatial leakage interferences (Colclough, Brookes, Smith, \& Woolrich, 2015).

\section{Construction of AE-HMM over all subjects}

To further explore dynamics in whole brain, MEG data in source space were processed using matlab-embedded HMMAR toolbox (Vidaurre et al., 2016). In this study, an AE-HMM was constructed to exhibit the process of dynamics transition during the emotional face task. Before estimating the model, whole-brain voxel set in source space were projected onto ninety brain regions based on AAL90 template. Subsequently, source time-series were filtered between $1-120 \mathrm{~Hz}$ and the amplitude 
envelope was calculated using Hilbert transition. Data were concatenated across all individuals to constitute a big 3-dimension matrix whose first dimension is the number of individuals, and the second dimension is the number of brain regions, the third dimension is the number of time series. A schematic of the whole process was exhibited in Figure1.

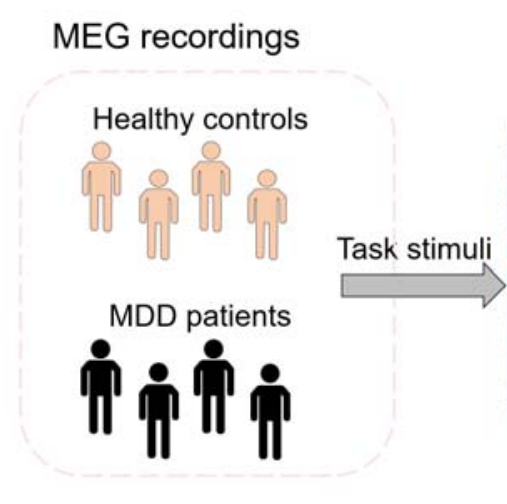

Hidden markov model outputs
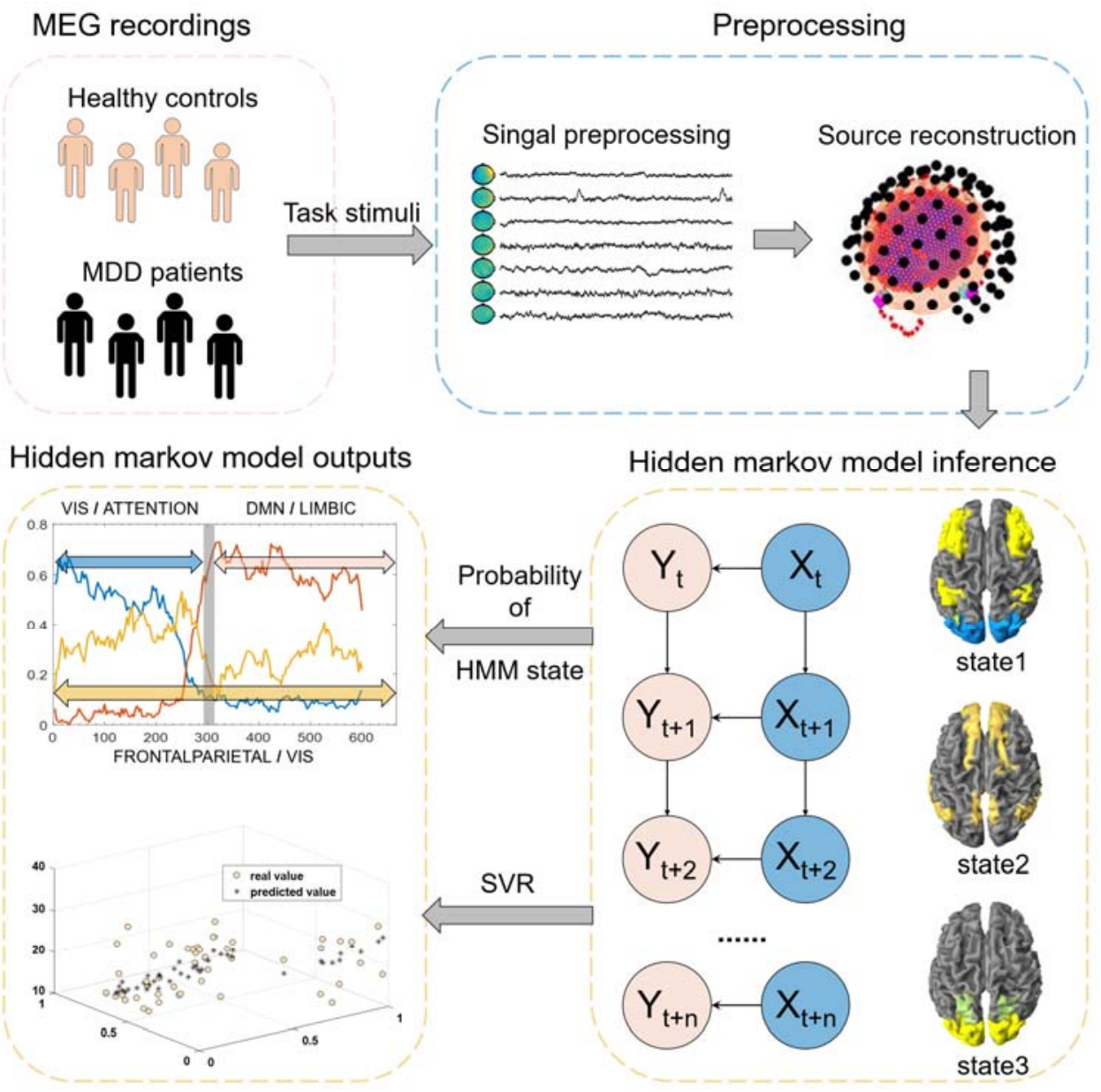

Hidden markov model inference

Figure 1 The architecture of the whole pipeline

(A) A schematic for the preprocessing of MEG signal applied prior to HMM analysis. (B) A schematic for HMM model, in which $X_{t}$ denotes the brain resides at time point t, while the $Y_{t}$ denotes the observed data. (C) An illustration of brain activation patterns from HMM used to predict disorders severity with Support-Vector-Regression model. 
Considering the variance distribution of our data and making sure the lowest free energy, this study denoted $\mathrm{K}=3$ before training the model which also followed findings in Hirschmann's study (Hirschmann et al., 2020). The observation model subsequently was constructed by training 1000 iterations with pre-defined K states.

\section{Statistical analysis over state-wise spatial-temporal characters between MDDs} and HCs

To characterize the spatial power distribution of each state, covariance matrix was obtained from observation model. The mean activation map was calculated by averaging the prior distributions of the envelope value for brain regions in each state. According to the study in 2019 (Luppi et al., 2019), regions in AAL90 template were divided into seven networks based on dynamic interactions and diversified functions of brain. Each inferred state was shown together with a mean activation for distinct brain networks. Using the time courses of posterior probabilities inferred from HMM, the power activation map of each subject was estimated. Next, non-parametric two-sample $t$ tests were utilized over state-wise power activation in network level between MDD and HC groups. The multiple comparisons were controlled via a Bonferroni correction.

In addition, Fractional occupancy (FO) was computed to characterize the dynamics of inferred brain states in each participant. This descriptor is defined as the proportion of 
each state time spent in the whole time length (Zhi et al., 2018). To further analyze the dysfunction of brain dynamics, FO values in each state were then compared between MDDs and HCs by two-sample independent $t$ test.

\section{Correlational analysis between dynamic characters and MDD severity}

To assess the relation between patients' disease severity and HMM descriptors, a support vector machine regression (SVR) algorithm was applied to regress disease severity in MDD using FO values and HAM-D scores. A Gaussian kernel regression was applied with a leave-one-out-cross-validation (LOOCV). Mean Absolute Error (MAE) was then calculated by average absolute bias between predictive value and real value to assess the predictive performances. Finally, correlational analysis was performed between predicted scores and real HAM-D scores via Pearson correlation. 


\section{Results}

\section{State-wise temporal-spatial patterns for all subjects}

In current study, three brain states were identified by AE-HMM over all subjects. As observed in Figure2 A, the fractional occupancy of hidden states at each time point was exhibited through the whole time. Specifically, state1 has a sustained proportion after stimulus onset, lasting about $270 \mathrm{~ms}$ and characterizing the early stage of task. State 2 showed a large occupancy between 270 and 600ms during the middle and latter part of the epoch. State3 exhibited a relatively small but constant occupancy over the whole post-stimulus process.

In spatial view, as shown in Figure 2 B, only brain networks with top $30 \%$ activation level was retained to characterize the most active networks of each state. Specifically, state1 showed relatively active neural activation in visual network (VN) and dorsal attention network (DAN), while the brain activity in state2 was mainly occupied by limbic system and default mode network (DMN). State3 was featured with dominant brain activation in $\mathrm{VN}$ and fronto-parietal control network (FPN).
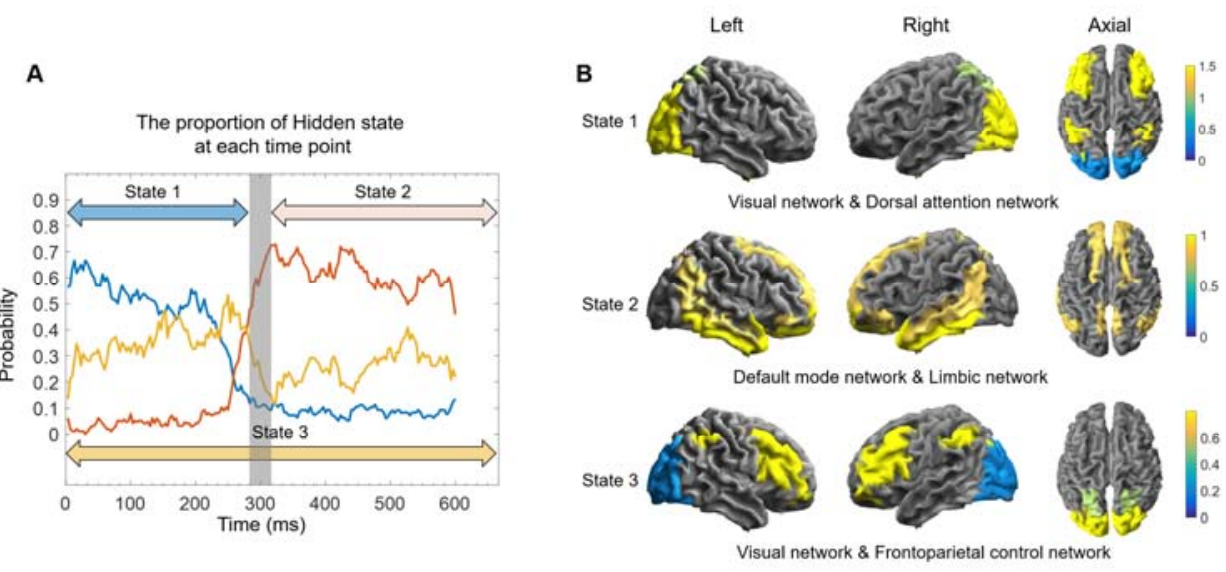


\section{Figure 2 The spatial-temporal brain states of all subjects}

(A) The averaged probability distribution during the pre-post stimuli across all subjects. The red

line denoting state1, pink line denoting state2, yellow line denoting state3. (B) The activation pattern characterized by different brain networks in three states. Time series data were normalized to have zero mean, and the regions of top $30 \%$ activation level were retained.

\section{Disrupted spatial-temporal patterns associated with MDD}

After comparing network power between MDD and HC groups, network with significant differences were found in two states: limbic system in state 2 and FPN in state3. Brain regions in these two networks were illustrated in Figure3 A, relative to HCs, MDD patients manifested significant increased brain activation in limbic system during state2 ( $p=0.0045$, survived after Bonferroni correction) and significant attenuated activity in FPN during state3 $\left(p=5.38 * 10^{-5}\right.$, survived after Bonferroni correction) (Figure3 B).

A
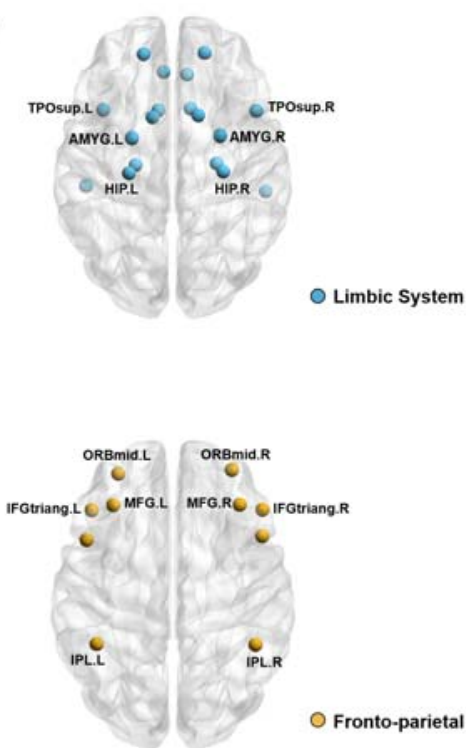
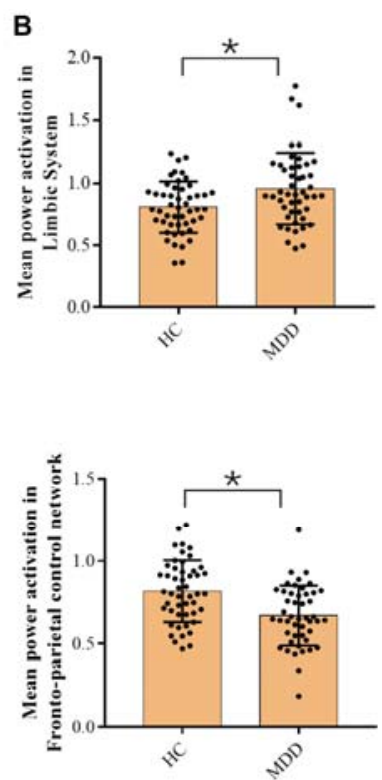
Figure 3 Aberrant spatial patterns for MDD versus HCs

(A) Individual network activation maps were compared between MDDs and HCs, and the regions in distinctive networks were shown. The blue nodes represented regions in limbic system and yellow nodes represent regions in FPN (the detailed contents of brain abbreviations in supplementary Table1) (B) The distribution of mean network power between HC and MDD groups. The above picture represented mean power activation in limbic system and the below picture represented mean power activation in FPN. * $p<0.05$ after Bonferroni correction.

For temporal characters, after comparing FO values of each state between $\mathrm{HC}$ and MDD groups, MDD subjects exhibited significant higher FO values in state1 $(p=$ 0.009, survived after Bonferroni correction) and lower FO values in state 2 versus HCs ( $p=0.006$, survived after Bonferroni correction) (Figure4 A).

\section{Dynamic characters related to MDD severity}

Since dysfunction of FO was proven to be associated with the depressed disorders, the descriptor was selected as the indicator to the severity of disorders in MDD group. As shown in Figure4 B, the spatial distribution of the real value of HAM-D total scores was consistent with predicted value visually. In statistical, the residuals for each depressed individual were exhibited in Figure4 C, satisfactory predicted outcomes, which meant that the residuals of MAE in the $95 \%$ confidence interval, were obtained in 45 subjects out of all the 47 individuals with MDD (Satisfactory ratio: 95.7\%). 
Furthermore, real HAM-D score across all depressed patients were significantly correlated with predicted outcomes in Figure4 D $(p=0.0062, \mathrm{r}=0.3933)$.

A

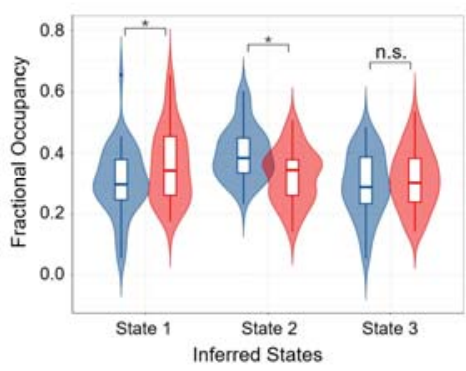

C

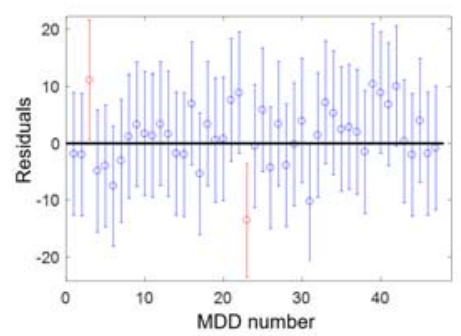

B

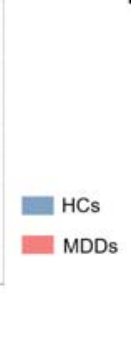

D
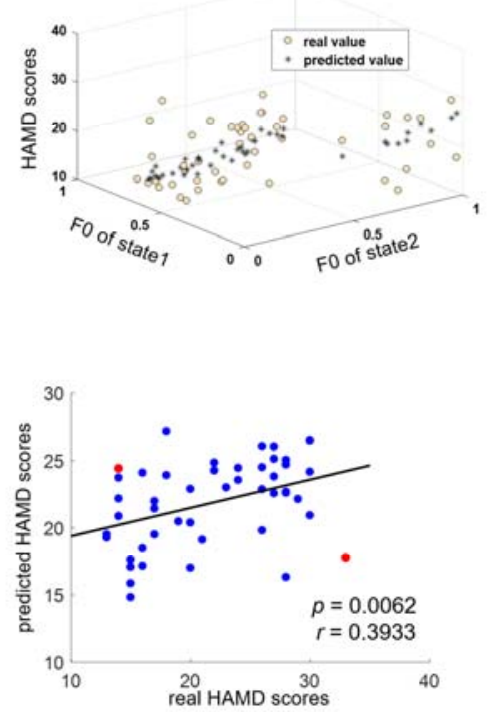

\section{Figure 4 Prediction of disorders severity using FO}

(A) The violins plot for the distributions of FO values in brain states between HC and MDD groups. ${ }^{*} p<0.05$ after Bonferroni correction, ns not significant. (B) The spatial distribution of clinical scores predicted by SVR model, $\mathrm{X}$ axes and $\mathrm{Y}$ axes represented FO values of visual state and limbic state respectively, and the magnitude of $\mathrm{Z}$ axes expressed the clinical scores, the black hollow dots representing real scores while the green asterisks denoted predicted scores. (C) Residuals distributions of predicted scores for each depressed individual, the blue line segment with a middle dot meant the residuals of MAE still in the $95 \%$ confidence interval, whereas the red line segment represented the MAE for this individual out of the confidence interval. (D) The Pearson correlation with predicted HAMD scores and real scores $(p=0.0062, \mathrm{r}=0.3933)$. 


\section{Discussion}

This study characterized the aberrant brain activation patterns of mood disorders and found specific state regulating different brain systems through diverse temporal stages of the sad facial recognition task. Besides, dynamic descriptors of FO inferred from each state were regarded as valuable index for individualized severity of depression.

First, the current study revealed the common neural patterns under the negative stimuli for all subjects. We inferred the whole procedure of negative emotions processing into three separate brain states. Specifically, during the early stage of visual stimuli processing (around $0-270 \mathrm{~ms}$ ), subjects showed dominant activation in VN and DAN. During the middle and latter processing stage after the onset of the stimuli (around 270-600ms), subjects manifested more activation in limbic system and DMN. Besides, subjects exhibited a relative small but essential proportion of brain activation in FPN and VN in the whole task $(0-600 \mathrm{~ms})$. These findings were consistent with what previous literature has reported in visual perception tasks (Quinn et al., 2018). In general, subjects may show an early occipital response after viewing the facial expressions and then gradually transform to the response to the elaborate emotional contents underlying faces by regulating limbic system.

Relative to HCs, MDD patients manifested the aberrant temporal-spatial pattern during the whole process. In early perceptual stage viewing sad expressions, depressed patients manifested statistically decreased FO value in early visual state 
contrast to HCs. The attenuated FO for early visual perception meant that MDDs spent shorter time capturing negative information from expressions, which suggested the rapid recognition toward sad faces, corresponding to negative emotions biases in previous findings (Almeida, Pajtas, Mahon, Nakayama, \& Caramazza, 2013; Sterzer, Hilgenfeldt, Freudenberg, Bermpohl, \& Adli, 2011). Besides, in line with previous findings, depressed patients who manifested decreased VN activity might affect visual perception function that was associated with the occurrence and recurrence of mental disorders (Dai \& Feng, 2011). Furthermore, previous evidence supported that the DAN often exerts a top-down regulation on the $\mathrm{VN}$ to integrate information during the visual perception stage. Thus, this aberrant brain state might represent the dysfunction of top-down mechanism for primary processing, which indicated that MDDs failed to treat negative information comprehensively and their inhibited visual-related cortices made them overexpress negative things and neglect other information (Desseilles et al., 2011; Schupp et al., 2004; Vossel, Geng, \& Fink, 2014). In middle and later stage for deep processing of emotional elements. Compared with HC group, depressed patients manifested significant increased FO values as well as hyper-activated power in limbic system of the state. Since limbic-related regions involved amygdala, hippocampus have been considered as the core for processing human expressions configuration and elaborate emotional contents (Almeida et al., 2013; Sterzer et al., 2011), significant higher FO values in limbic state meant that MDDs spent more time indulging in sad contents and over expressing or even ruminating in negative things. Certainly, this kind of aberrant dynamic character 
underpinned by the impaired pathological neural circuits. Current study reported that statistically elevated activity was observed in limbic system versus HCs during the middle and later stage. Combined with previous findings, we thought that negative information successfully elicited robust responsiveness on limbic system, especially core regions like amygdala (Hall et al., 2014; Suslow et al., 2010). Generally, hyper-activated limbic system in the deeper processing of negative information may be another potential indicator to depressive disorders.

Another important finding was that MDD patients exhibited statistically attenuated power activation of FPN relative to HCs during the whole process. The underlying mechanism underpinning the dysregulated FPN was essential for explaining the neural mechanism of MDD. Previous studies found that FPN was involved in highly adaptive control processes and suggested it could communicate with other systems throughout the brain (Power et al., 2011). In other words, the FPN might regulate other networks to coordinate with specific goals and reduce the goal-disrupting effects brought by other networks. Specifically, evidence supported that both DAN coordinating attention to task stimuli, and limbic system connecting to mental states may be regulated by FPN (Cole, Repovs, \& Anticevic, 2014). In present exploratory analyses, although there existed no statistically difference in temporal character between MDDs and HCs, decreased activity of FPN was found in MDDs contrast to HCs. Hypo-activated FPN state may interfere the coordinating function that influences goal-driven brain activities and result in dysregulated brain functional 
networks. The disrupted spatial patterns of FPN state could also be viewed as a potential pathophysiological biomarker for identifying depression.

Besides, in current exploratory analyses, the FO values of early visual and limbic states were applied as input features of a regression model, which get the predicted outcomes consistent with real HAM-D scores. This finding meant that simple FO parameters describing the aberrant distribution of HMM state occupancies could predict the specific disorder severity for each subject. The satisfactory prediction results of $95.7 \%$ may be resulted from the process as we descried above that depressed patients perceive the negative emotion from the stimuli more quickly and then over experience in the sad contents. The overall dysfunction for the distort time allocation implied the defective emotional regulation system underpinned by the impaired neural circuits of MDD. Thus, the dynamic character of FO values could not only discriminate the MDD patients but also indicate the individual depression severity.

In summary, we reconstructed the brain states in the overall process of a passively sad expression recognition task via AE-HMM and found that aberrant temporal-spatial patterns in different process stages like primary visual processing and emotional contents processing were correspond to the different dysfunction of brain functions for MDD respectively. Furthermore, dynamic descriptors of FO values inferred from HMMs could reflect the aberrant dynamism and predict the severity of MDD 
precisely. Overall, our findings may offer new insights on the pathology of negative emotion process for MDD and predict individual depression severity via a very simple dynamic character. 


\section{References}

Almeida, J., Pajtas, P. E., Mahon, B. Z., Nakayama, K., \& Caramazza, A. (2013). Affect of the unconscious: visually suppressed angry faces modulate our decisions. Cogn Affect Behav Neurosci, 13(1), 94-101. doi: 10.3758/s13415-012-0133-7

Bi, K., Hua, L., Wei, M., Qin, J., Lu, Q., \& Yao, Z. (2016). Dynamic functional-structural coupling within acute functional state change phases: Evidence from a depression recognition study. $J$ Affect Disord, 191, 145-155. doi: 10.1016/j.jad.2015.11.041

Bi, K., Luo, G., Tian, S., Zhang, S., Liu, X., Wang, Q., . . . Yao, Z. (2019). An enriched granger causal model allowing variable static anatomical constraints. Neuroimage Clin, 21, 101592. doi: 10.1016/j.nicl.2018.11.002

Charquero-Ballester, M., Kleim, B., Vidaurre, D., Ruff, C., Stark, E., Tuulari, J. J., . . Ehlers, A. (2020). Effective psychological treatment for PTSD changes the dynamics of specific large-scale brain networks. Preprint. doi: 10.1101/2020.01.07.891986

Colclough, G. L., Brookes, M. J., Smith, S. M., \& Woolrich, M. W. (2015). A symmetric multivariate leakage correction for MEG connectomes. Neuroimage, 117, 439-448. doi: 10.1016/j.neuroimage.2015.03.071

Cole, M. W., Repovs, G., \& Anticevic, A. (2014). The frontoparietal control system: a central role in mental health. Neuroscientist, 20(6), 652-664. doi: 10.1177/1073858414525995

Cribben, I., Haraldsdottir, R., Atlas, L. Y., Wager, T. D., \& Lindquist, M. A. (2012). Dynamic connectivity regression: Determining state-related changes in brain connectivity. Neuroimage, 61(4), 907---920.

Dai, Q., \& Feng, Z. (2011). Deficient interference inhibition for negative stimuli in depression: an event-related potential study. Clin Neurophysiol, 122(1), 52-61. doi: 10.1016/j.clinph.2010.05.025

Dalili, M. N., Penton-Voak, I. S., Harmer, C. J., \& Munafo, M. R. (2015). Meta-analysis of emotion recognition deficits in major depressive disorder. Psychol Med, 45(6), 1135-1144. doi: 10.1017/S0033291714002591

Desseilles, M., Schwartz, S., Dang-Vu, T. T., Sterpenich, V., Ansseau, M., Maquet, P., \& Phillips, C. (2011). Depression alters "top-down" visual attention: a dynamic causal modeling comparison between depressed and healthy subjects. Neuroimage, 54(2), 1662-1668. doi: 10.1016/j.neuroimage.2010.08.061

Diego, V., Hunt, L. T., Quinn, A. J., Hunt, B. A. E., Brookes, M. J., Nobre, A. C., \& Wool rich, M. W. (2018). Spontaneous cortical activity transiently organises into frequency specific phase-coupling networks. Nat Commun, 9(1), 2987-.

Furey, M. L. (2011). The prominent role of stimulus processing: cholinergic function and dysfunction in cognition. Curr Opin Neurol, 24(4), 364-370. doi: 10.1097/WCO.0b013e328348bda5

Furey, M. L., Drevets, W. C., Hoffman, E. M., Frankel, E., Speer, A. M., \& Zarate, C. A., Jr. (2013). Potential of pretreatment neural activity in the visual cortex during emotional processing to predict treatment response to scopolamine in major depressive disorder. JAMA Psychiatry, 70(3), 280-290. doi: 10.1001/2013.jamapsychiatry.60

Hall, L. M., Klimes-Dougan, B., Hunt, R. H., Thomas, K. M., Houri, A., Noack, E., . . Cullen, K. R. (2014). An fMRI study of emotional face processing in adolescent major depression. J Affect Disord, 168, 44-50. doi: 10.1016/j.jad.2014.06.037

Hirschmann, J., Baillet, S., Woolrich, M., Schnitzler, A., Vidaurre, D., \& Florin, E. (2020). Spontaneous 
network activity $<35 \mathrm{~Hz}$ accounts for variability in stimulus-induced gamma responses. Neuroimage, 207, 116374. doi: 10.1016/j.neuroimage.2019.116374

Jiang, H., Hua, L., Dai, Z., Tian, S., Yao, Z., Lu, Q., \& Popov, T. (2019). Spectral fingerprints of facial affect processing bias in major depression disorder. Soc Cogn Affect Neurosci, 14(11), 1233-1242. doi: 10.1093/scan/nsz096

Jing-Lun, D. U., Yao, Z. J., \& Xie, S. P. J. C. M. H. J. (2007). The Primary Development of Chinese Facial Expression Video System. 21(5), 333-337.

Jung, T.-P., Makeig, S., Westerfield, M., Townsend, J., \& Sejnowski, T. J. (2001). Analysis and visualization of single-trial event-related potentials. Hum Brain Mapp, 14(3), 166-185.

Luppi, A. I., Craig, M. M., Pappas, I., Finoia, P., Williams, G. B., Allanson, J., .. Stamatakis, E. A. (2019). Consciousness-specific dynamic interactions of brain integration and functional diversity. Nat Commun, 10(1), 4616. doi: 10.1038/s41467-019-12658-9

Mel'Nikov, M. E., Petrovskii, E. D., Bezmaternykh, D. D., Kozlova, L. I., Natarova, K. A., \& Medicine. (2018). fMRI Response of Parietal Brain Areas to Sad Facial Stimuli in Mild Depression. $J$ Bulletin of Experimental Biology Medicine, 165(5), 361-365.

Oostenveld, R., Fries, P., Maris, E., \& Schoffelen, J. M. (2011). FieldTrip: Open source software for advanced analysis of MEG, EEG, and invasive electrophysiological data. Comput Intell Neurosci, 2011, 156869. doi: 10.1155/2011/156869

Power, J. D., Cohen, A. L., Nelson, S. M., Wig, G. S., Barnes, K. A., Church, J. A., . . Schlaggar, B. L. J. N. (2011). Functional network organization of the human brain. Neuron, 72(4), 665-678.

Quinn, A. J., Vidaurre, D., Abeysuriya, R., Becker, R., Nobre, A. C., \& Woolrich, M. W. (2018). Task-Evoked Dynamic Network Analysis Through Hidden Markov Modeling. Front Neurosci, 12, 603. doi: $10.3389 /$ fnins.2018.00603

Roiser, J. P., Elliott, R., \& Sahakian, B. J. (2012). Cognitive mechanisms of treatment in depression. Neuropsychopharmacology, 37(1), 117-136. doi: 10.1038/npp.2011.183

Schupp, H. T., Ohman, A., Junghofer, M., Weike, A. I., Stockburger, J., \& Hamm, A. O. (2004). The facilitated processing of threatening faces: an ERP analysis. Emotion, 4(2), 189-200. doi: 10.1037/1528-3542.4.2.189

Soni, S., Muthukrishnan, S. P., Sood, M., Kaur, S., \& Sharma, R. (2018). Hyperactivation of left inferior parietal lobule and left temporal gyri shortens resting EEG microstate in schizophrenia. Schizophr Res, 201, 204-207. doi: 10.1016/j.schres.2018.06.020

Sterzer, P., Hilgenfeldt, T., Freudenberg, P., Bermpohl, F., \& Adli, M. (2011). Access of emotional information to visual awareness in patients with major depressive disorder. Psychol Med, 41(8), 1615-1624. doi: 10.1017/\$0033291710002540

Stuhrmann, A., Suslow, T., \& Dannlowski, U. (2011). Facial emotion processing in major depression: a systematic review of neuroimaging findings. Biol Mood Anxiety Disord, 1(1), 10. doi: 10.1186/2045-5380-1-10

Suslow, T., Konrad, C., Kugel, H., Rumstadt, D., Zwitserlood, P., Schoning, S., . . Dannlowski, U. (2010). Automatic mood-congruent amygdala responses to masked facial expressions in major depression. Biol Psychiatry, 67(2), 155-160. doi: 10.1016/j.biopsych.2009.07.023

Vidaurre, D., Quinn, A. J., Baker, A. P., Dupret, D., Tejero-Cantero, A., \& Woolrich, M. W. (2016). Spectrally resolved fast transient brain states in electrophysiological data. Neuroimage, 126, 81-95. doi: 10.1016/j.neuroimage.2015.11.047 
Vossel, S., Geng, J. J., \& Fink, G. R. (2014). Dorsal and ventral attention systems: distinct neural circuits but collaborative roles. Neuroscientist, 20(2), 150-159. doi: 10.1177/1073858413494269

Watters, A. J., Harris, A. W. F., \& Williams, L. M. (2018). Electrocortical reactivity to negative and positive facial expressions in individuals with a family history of major depression. Biol Psychol, 136, 127-135. doi: 10.1016/j.biopsycho.2018.05.015

Woolrich, M., Hunt, L., Groves, A., \& Barnes, G. (2011). MEG beamforming using Bayesian PCA for adaptive data covariance matrix regularization. Neuroimage, 57(4), 1466-1479. doi: 10.1016/j.neuroimage.2011.04.041

Zhang, S., Tian, S., Chattun, M. R., Tang, H., \& Lu, Q. (2018). A supplementary functional connectivity microstate attached to the default mode network in depression revealed by resting-state magnetoencephalography. Progress in Neuro Psychopharmacology \& Biological Psychiatry, $83,76$.

Zhi, D., Calhoun, V. D., LV, L., Ma, X., \& Ke, Q. (2018). Aberrant Dynamic Functional Network Connectivity and Graph Properties in Major Depressive Disorder. Paper presented at the International Conference of the IEEE Engineering in Medicine \& Biology Society. 